\title{
Indicadores socioambientais de sistemas agroflorestais no Cerrado Goiano
}

O estudo propõe e analisa indicadores socioambientais de sistemas agroflorestais (SAFs) multiestrata inseridos no Cerrado goiano, para tanto, emprega o Marco para Evaluación de Sistemas de Manejo de Recursos Naturales Incorporando Indicadores de Sustentabilidad (MESMIS). É proposto ainda um índice socioambiental geral (ISG) para cada um dos oito SAFs analisados, bem como uma análise de agrupamento hierárquico para verificar as similaridades ou as diferenças entre os sistemas com base nos indicadores. Com a adaptação do método MESMIS foi possível observar a eficiência produtiva dos SAFs e dos agroecossistemas, bem como alguns fatores que influenciam no potencial de comercialização dos produtos oriundos desses sistemas. Foi possível identificar ainda, os perfis de parte dos agricultores que cultivam SAFs no Cerrado goiano, um pouco das características organizacionais dos agroecossistemas, também qual a contribuição desses sistemas para a qualidade de vida dos agricultores.

Palavras-chave: Agroecossistemas; Agrofloresta; MESMIS; Agroecologia; Bioma Cerrado.

\section{Socio-environmental indicators of agroforestry systems in the Cerrado of Goiás}

\begin{abstract}
The study proposes and analyzes socio-environmental indicators of multi-stratified agroforestry systems (AFS) inserted in the Cerrado of Goiás, for this purpose, it uses the Framework for Marco para Evaluación de Sistemas de Manejo de Recursos Naturales Incorporando Indicadores de Sustentabilidad (MESMIS). A general socio-environmental index (ISG) is also proposed for each of the eight AFS analyzed, as well as a hierarchical cluster analysis to verify the similarities or differences between the systems based on the indicators. With the adaptation of the MESMIS method, it was possible to observe the productive efficiency of AFS and agroecosystems, as well as some factors that influence the commercialization potential of products from these systems. It was also possible to identify the profiles of part of the farmers that cultivate AFS in the Cerrado of Goiás, a little of the organizational characteristics of the agroecosystems, also what is the contribution of these systems to the quality of life of the farmers.
\end{abstract}

Keywords: Agroecosystems; Agroforestry; MESMIS; Agroecology; Cerrado biome.

\section{Topic: Agroecologia}

Reviewed anonymously in the process of blind peer.
Received: 01/01/2021

Approved: $25 / 01 / 2021$
Leovigildo Aparecido Costa Santos (iD Universidade Estadual de Goiás, Brasil http://lattes.cnpq.br/4377455906108645 http://orcid.org/0000-0002-5768-8606 eng.leovigildo@gmail.com

Wander Gomes de Souza (iD

Universidade Federal dos Vales do Jequitinhonha e Mucuri, Brasil http://lattes.cnpq.br/2182017526367163 http://orcid.org/0000-0002-4341-8090 wander.g.souza@live.com

Murilo Mendonça Oliveira de Souza (iD) Universidade Estadual de Goiás, Brasil http://lattes.cnpq.br/0747751464696905 http://orcid.org/0000-0003-4686-9388 murilosouza@hotmail.com
Carlos de Melo Silva Neto id Instituto Federal de Goiás, Brasil http://lattes.cnpq.br/2681735180356106 http://orcid.org/0000-0001-8624-3836 carloskoa@gmail.com
Referencing this:

SANTOS, L. A. C.; SOUZA, W. G.; SOUZA, M. M. O.; SILVA NETO, C. M. Indicadores socioambientais de sistemas agroflorestais no Cerrado Goiano. Revista Ibero Americana de Ciências Ambientais, v.12, n.1, p.54-65, 2021. DOI: http://doi.org/10.6008/CBPC21796858.2021.001.0006

DOI: 10.6008/CBPC2179-6858.2021.001.0006 


\section{INTRODUÇÃO}

Grande parte do Cerrado já foi transformada em pasto e agricultura para expansão do agronegócio. A conversão agrícola para a soja e a criação de gado em larga escala, juntamente à contaminação do solo, água e ar por agrotóxicos, adubos e outros corretivos químicos, representam a maior ameaça ao bioma (FERNANDES et al., 2011).

Em oposição ao agronegócio, a agroecologia emerge no país como um movimento social que propõe práticas agrícolas alternativas, se constituindo como uma ciência integradora que interage com os conhecimentos locais e com diversas outras ciências (FONTORA et al., 2016). Não se limitando apenas às mudanças tecnológicas ou a uma simples ecologização da agricultura, as propostas elaboradas no âmbito da agroecologia vão muito além das questões ambientais, abrangendo também os aspectos econômicos, sociais e políticos do desenvolvimento agrícola (SILVA NETO, 2014).

Nesse sentido, dentre as muitas práticas que integram a produção agroecológica estão os sistemas agroflorestais (SAFs). São sistemas de produção milenares, desenvolvidos em diferentes regiões do planeta (NAIR, 1991; NAIR, 1993; PENEIREIRO et al., 2002), mas somente há algumas décadas a ciência tem se dedicado a estudar seus benefícios e custos, assim como as complexas interações que ocorrem entre seus componentes vegetais, animais e os seres humanos (MICCOLIS et al., 2016).

O principal objetivo dos SAFs é a otimização do uso da terra, conciliando a produção de alimentos, energia e serviços ecossistêmicos com a produção florestal, diminuindo à pressão pelo uso da terra na agropecuária convencional e possibilitando a conservação do potencial produtivo do solo, por meio de sistemas agroecológicos mais estáveis (DUBOC, 2008).

Nas últimas décadas tem crescido o interesse de avaliação da sustentabilidade e dos benefícios proporcionados tanto ao ambiente, quanto aos seres humanos por sistemas agrícolas agroecológicos (SANTAMARIA et al., 2017). Desta forma, a avaliação de indicadores socioambientais dos sistemas agroflorestais multiestrata é fundamental para verificar a eficiência da conversão de um modelo de produção convencional para uma produção com base nos princípios agroecológicos. Diante disso, os objetivos desse estudo foram a proposição e aplicação de um conjunto de indicadores socioambientais para sistemas agroflorestais do Cerrado goiano empregando se o Marco para Evaluación de Sistemas de Manejo de Recursos Naturales Incorporando Indicadores de Sustentabilidad (MESMIS).

\section{MATERIAIS E MÉTODOS}

\section{Método de análise}

O método empregado para análise foi o MESMIS, por apresentar alta adaptabilidade a diferentes sistemas produtivos e por abranger vários atributos do agroecossistema (produtividade, estabilidade, adaptabilidade, autossuficiência e resiliência), que podem ser avaliados através de diversos critérios diagnósticos, por meio dos quais se propõe os indicadores (MASERA et al., 2000).

O MESMIS é constituído por uma estrutura cíclica, pois utiliza uma série de etapas que conduzem à 
reavaliação do sistema. Ele apresenta certa flexibilidade para se adaptar aos diferentes níveis de informação (qualitativa ou quantitativa) e capacidades técnicas, partindo de uma abordagem participativa que facilita a promoção de discussões e o alcance de respostas entre avaliadores e avaliados (MONTELLANO, 2013).

Para caracterização a aplicação do MESMIS, o primeiro passo é a delimitação do agroecossistema e seus subsistemas, identificando e caracterizando o sistema de produção alvo do estudo, bem como suas relações com outros elementos do agroecossistema (núcleo gestor, demais subsistemas produtivos, recursos naturais, etc.). A etapa seguinte consiste em definir indicadores para avaliar os dados levantados na etapa anterior, para tanto, são elaborados os atributos de temáticas generalistas (produtividade, equidade, estabilidade, etc.) a depender das características e do contexto socioeconômico e ambiental do agroecossistema.

Os critérios de diagnósticos traduzem cada um dos atributos em elementos mais concretos e representam uma ponte para a definição dos indicadores. Posteriormente os indicadores são sintetizados em matrizes, onde há a atribuição e padronização dos pesos para cada um, também suas representações gráficas e interpretações (Figura 1).

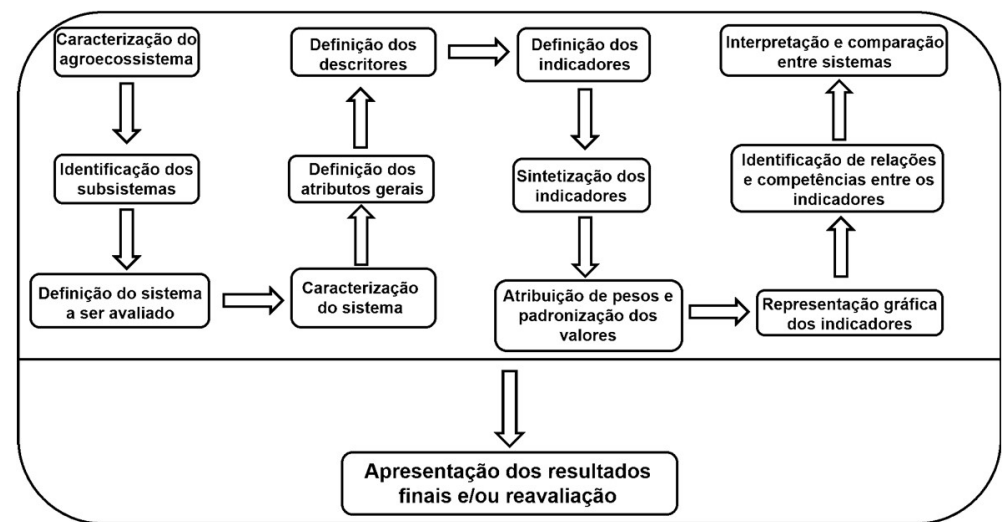

Figura 1: Fluxograma de execução do método MESMIS. Fonte: Adaptado de Masera et al. (2000).

\section{Sistemas agroflorestais estudados}

Foram estudados oito sistemas agroflorestais, distribuídos por seis municípios Goianos: Niquelândia, Ceres, Aragoiânia, Hidrolândia, Cristianópolis e Ipameri. Os SAFs são semelhantes no que diz respeito à baixa utilização de insumos externos aos agroecossistemas, principalmente agroquímicos, sendo que em somente um sistema foi observado o uso de adubação química por NPK. O tempo de implantação varia entre dois e doze anos, área ocupada entre $400 \mathrm{~m}^{2}$ (0,04 ha) e $18.000 \mathrm{~m}^{2}$ (1,8 ha) (Tabela 1).

Tabela 1: Informações sobre os SAFs multiestrata estudados: área ocupada $\left(\mathrm{m}^{2}\right)$, tamanho da propriedade (hectares), e idade de implantação.

\begin{tabular}{lllll}
\hline SAF & Município & Área por SAFs $\left.\mathbf{( m}^{\mathbf{2}}\right)$ & Total da propriedade $($ ha) & Idade (anos) \\
\hline SAF01 & Ceres & 800 & 200 & 12 \\
SAF02 & Ceres & 12.000 & 3,6 & 4 \\
SAF03 & Hidrolândia & 400 & 42,5 & 3 \\
SAF04 & Aragoiânia & 18.000 & 100 & 3 \\
SAF05 & Cristianópolis & 800 & 3 & 2 \\
SAF06 & Cristianópolis & 800 & 61,5 & 9 \\
SAF07 & Ipameri & 5.000 & 28,5 & 2 \\
SAF08 & Niquelândia & 15.000 & 114 & 4 \\
\hline
\end{tabular}


Com base nas classificações propostas por Nair (1985) todos os SAFs estudados são agrossilviculturais, com diferentes combinações de componentes arbóreo com culturas agrícolas, sem a criação de animais na mesma área. Ambos são multiestrata, que de acordo com Schroth et al. (2014) é o tipo de sistema agroflorestal mais cultivado em regiões tropicais. Esse tipo de SAF apresenta como principal característica a configuração do dossel em múltiplos andares, ou estratos, como ocorre nos sistemas florestais naturais.

Com base nos arranjos de seus componentes, os sistemas são coincidentes ou simultâneos, com as culturas anuais, semiperenes e perenes cultivadas em combinação com espécies madeireiras ou de uso múltiplo. Quanto ao arranjo espacial, são densos, e conforme a definição de Nair (1985) apresentam diversidade de espécies e fornecimento de múltiplos produtos.

Dois dos SAFs estudados estão inseridos em instituições de ensino, um no Instituto Federal Goiano do município de Ceres (SAF01) e, outro no Instituto Tiradentes (SAF08), uma escola de ensino técnico no município de Niquelândia. Na maioria dos agroecossistemas o núcleo gestor é formado por pessoas com nível superior de escolaridade, apenas um SAF se encontra em assentamento rural (SAF07), em Ipameri, sendo o único onde o núcleo gestor é composto por pessoas com o nível médio de escolaridade.

\section{Obtenção dos dados}

Os dados para sintetização dos indicadores foram gerados com base na caracterização ambiental dos SAFs, envolvendo as composições florísticas arbórea e agrícola, bem como algumas características do solo como pH e teor de matéria orgânica. Também foram incorporadas na análise as informações referentes aos agroecossistemas onde os SAFs estão inseridos.

A flora foi caracterizada por meio de inventários florísticos, empregando-se o método do caminhamento, proposto por Filgueiras et al. (1994), com mensuração dos parâmetros de altura total e diâmetro na altura do peito ( $\mathrm{DAP}=1,3 \mathrm{~m}$ de altura) dos indivíduos arbóreos com circunferência na altura do peito (CAP) $\geq 15 \mathrm{~cm}$. Com os dados obtidos calculou-se os parâmetros fitossociológicos de riqueza e frequência das espécies arbóreas e culturas agrícolas.

Os indivíduos arbóreos foram identificados, quando possível até espécie, em campo ou com auxílio de literatura especializada como o Herbário Virtual Reflora (REFLORA, 2016), também com auxílio de especialistas. A identificação das culturas agrícolas foi feita em campo pelo nome comum juntamente com os(as) agricultores(as) e, posteriormente, ao nível de espécies.

As amostras de solo para análise foram coletadas em profundidade de $0-20 \mathrm{~cm}$ durante a estação chuvosa, entre os meses de janeiro e abril do ano de 2018. Em cada SAF foi obtida uma amostra composta, formada por três amostras simples coletadas aleatoriamente, o mesmo procedimento foi realizado em áreas de pastagem próximas. As análises seguiram os métodos propostos pela Embrapa (2011) e foram realizadas em laboratório credenciado pelo Ministério da Agricultura.

A caracterização dos agroecossistemas se deu através da aplicação de entrevistas semiestruturadas (conforme a proposição de TESSIER et al., 2018) e observação do pesquisador em campo, com posterior 
análise e proposição dos indicadores socioambientais. Os indicadores utilizados foram definidos com base em publicações de temática semelhante, adaptados ao contexto da presente pesquisa (por exemplo, TORQUEBIAU, 1992; DANIEL, 2000; COSTA et al., 2012; NASCIMENTO et al., 2018).

\section{Definição dos indicadores}

Os indicadores foram definidos de forma generalista, de modo a abranger as características principais dos sistemas agroflorestais visitados, englobando os fatores sociais e ambientais inerentes aos SAFs, ao agroecossistema e ao contexto organizacional do núcleo gestor. A abordagem generalista visa garantir a reprodutibilidade para outros sistemas que apresentem semelhanças estruturais e de manejo agroecológico. A categorização dos indicadores foi baseada nas proposições do método de análise escolhido.

Não foram abordados os aspectos econômicos dos SAFs, como o custo de implantação, renda exata obtida com a venda dos produtos, produção total e comercialização de cada componente, bem como os custos de manutenção, isso por serem variáveis que demandam um maior tempo de acompanhamento do agroecossistema e por não ser uma das metas do estudo. Desta forma, foram levados em consideração os aspectos referentes à qualidade de vida, alimentação, trabalho, nível organizacional e o contexto social das pessoas que se relacionam com o agroecossistema, bem como a qualidade e conservação dos recursos naturais inseridos nele e suas relações com o meio externo.

Ao todo, 45 indicadores foram desenvolvidos, inseridos em vinte descritores e cinco atributos, quatro deles usuais no MESMIS e um adaptado à situação do presente estudo.

No atributo 'Produtividade' os indicadores refletem a eficiência e produção do SAF e do agroecossistema. No atributo 'Estabilidade, resiliência e confiabilidade' refletem a diversificação do SAF, as características ambientais, políticas e organizacionais do agroecossistema, bem como o potencial de comercialização dos produtos oriundos do sistema. Em 'Equidade' são indicadas as características referentes ao trabalho, nível de escolaridade e a relação entre o núcleo gestor do agroecossistema com outros agricultores. 'Em melhorias na vida humana' estão os fatores relacionados à contribuição do SAF para a qualidade de vida e saúde das pessoas. 'Autogestão' engloba os indicadores referentes à autonomia do agroecossistema em aproveitar os recursos locais e dispor de infraestrutura básica de moradia, equipamentos e instalações necessárias para garantir a produção (Quadro 1).

Quadro 1: quadro de indicadores socioambientais para sistemas agroflorestais no Cerrado goiano. Adaptado do método MESMIS (MASERA et al., 2000).

\begin{tabular}{|c|c|c|c|}
\hline $\begin{array}{l}\text { Atributos } \\
\text { Gerais }\end{array}$ & Descritores & Indicadores* & Medidas (pesos) \\
\hline \multirow{4}{*}{ 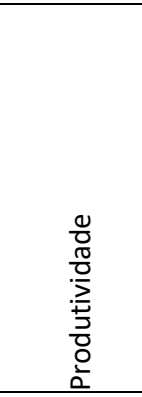 } & \multirow[b]{2}{*}{ Eficiência } & O SAF contribui para economia familiar & $\operatorname{Sim}(1)$, não $(0)$ \\
\hline & & $\begin{array}{l}\text { Participação do SAF nos rendimentos da } \\
\text { propriedade }\end{array}$ & Baixa (1), média (2), alta (3) \\
\hline & \multirow[t]{2}{*}{ Produção } & $\begin{array}{l}\% \text { de culturas agrícolas em relação ao total de } \\
\text { plantas do SAF }\end{array}$ & $\begin{array}{l}0-1 \%(0) \\
1,1-25 \%(1) \\
25,1-50 \%(2) \\
50,1-75 \%(3) \\
75,1-100 \%(4) \\
\end{array}$ \\
\hline & & Principais produtos do sistema & $\begin{array}{l}\text { Olericultura (1), fruticultura }(1) \text {, } \\
\text { silvicultura }(1) \text {, duas opções }(2) \text {, três }\end{array}$ \\
\hline
\end{tabular}




\begin{tabular}{|c|c|c|c|c|}
\hline & & & & opções (3). \\
\hline & & & Certificação de produção orgânica & $\operatorname{Sim}(1)$, não (0) \\
\hline & & & Diversificação produtiva do agroecossistema & $\begin{array}{l}\text { Um uso (1), dois usos (2), três usos (3), } \\
\text { quatro ou mais (4) }\end{array}$ \\
\hline & & & A terra é a única fonte de renda & $\operatorname{Sim}(1)$, não (0) \\
\hline & & & Qual a principal fonte de renda & Externa (1), agroecossistema (2) \\
\hline & & & Tamanho do SAF em relação à propriedade & $\begin{array}{l}\text { (Área do SAF x 100) / (Área do } \\
\text { agroecossistema) }\end{array}$ \\
\hline & & & Pretensão de expansão do SAF & $\operatorname{Sim}(1)$, não (0) \\
\hline & & & Pretensão de implantar outro SAF & $\operatorname{Sim}(1)$, não (0) \\
\hline & Diversidade & no & $\begin{array}{l}\text { \% de arbóreas nativas no SAF em relação ao } \\
\text { total de arbóreas }\end{array}$ & $\begin{array}{l}0-1 \%(0) \\
1,1-25 \%(1) \\
25,1-50 \%(2) \\
50,1-75 \%(3) \\
75-100 \%(4)\end{array}$ \\
\hline & tempo e espaço & & $\begin{array}{l}\text { Conectividade entre fragmentos florestais } \\
\text { pelos SAFs }\end{array}$ & $\begin{array}{l}\text { Até } 200 \mathrm{~m}(1) \\
\text { Até } 100 \mathrm{~m}(2) \\
\text { Até } 50 \mathrm{~m}(3)\end{array}$ \\
\hline & & & $\begin{array}{l}\text { Alimentos plantados nos SAFs (anuais, } \\
\text { bianuais, perenes) }\end{array}$ & $\begin{array}{l}\text { Anuais (1), bianuais (1), perenes (1), duas } \\
\text { das opções (2), as três (3) }\end{array}$ \\
\hline & & & Regularidade de APPs e RLs & $\begin{array}{l}\text { APP regular (1), } \\
\text { RL regular (1). } \\
\text { As duas opções (2) }\end{array}$ \\
\hline & & & Medidas conservacionistas no SAF & $\begin{array}{l}\text { Solo coberto com MO (1), curvas de nível } \\
\text { (1), as duas opções (2) }\end{array}$ \\
\hline & & & Medidas contra fatores de degradação & $\operatorname{Sim}(1)$, não (0) \\
\hline & Conservação & de & $\begin{array}{l}\text { Teor de matéria orgânica (MO) do solo em } \\
\text { relação a outro uso (ex.: pastagem) }\end{array}$ & Menor (0), igual (1), maior (2) \\
\hline & & & pH do solo: interpretação agronômica & $\begin{array}{l}\text { Muito baixo (0), muito alto (0). Baixo (1), } \\
\text { alto (1), bom (2). }\end{array}$ \\
\hline & & & Uso de adubos químicos & $\operatorname{Sim}(0)$, não (1) \\
\hline & & & Melhorias ambientais & $\begin{array}{l}\text { Solo (1), água (1), presença de fauna } \\
\text { nativa (1), diminuição do ataque de pragas } \\
\text { e doenças (1). Duas opções (2), três } \\
\text { opções (3) todas as opções (4) }\end{array}$ \\
\hline & & & Ataque de pragas & $\begin{array}{l}\text { Diminuiu (2), não notou diferenças (1), } \\
\text { aumentou (0) }\end{array}$ \\
\hline & & & Controle de pragas & Orgânico (1), químico (0) \\
\hline & sistema & do & Dificuldades enfrentadas & $\begin{array}{l}\text { Assistência técnica (4), manejo (4), } \\
\text { aquisição de insumos (4), comercialização } \\
\text { (4). Duas opções (3), três opções (2), todas } \\
\text { (1). }\end{array}$ \\
\hline & & & $\begin{array}{l}\text { Grau de acesso a políticas de compras } \\
\text { governamentais }\end{array}$ & $\begin{array}{l}\text { Sim (2), participou e não participa mais (1), } \\
\text { não (0). }\end{array}$ \\
\hline & $\begin{array}{l}\text { Políticas, } \\
\text { associativismo }\end{array}$ & & $\begin{array}{l}\text { Assistência técnica institucional ou } \\
\text { governamental }\end{array}$ & $\operatorname{Sim}(1)$, não (0) \\
\hline$\frac{0}{\frac{0}{0}}$ & movimentos & & $\begin{array}{l}\text { Participa ou apoia algum movimento social do } \\
\text { campo }\end{array}$ & $\operatorname{Sim}(1)$, não (0) \\
\hline$\overline{\overline{0}}$ & & & Participa de associação & $\operatorname{Sim}(1)$, não (0) \\
\hline 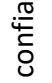 & & & Acesso à feiras e mercados & $\begin{array}{l}\text { Feiras (1), supermercado (1), feiras e } \\
\text { supermercados (2) }\end{array}$ \\
\hline 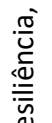 & Comercializacão & & Proximidade da cidade mais próxima & $\begin{array}{l}200 \mathrm{~km} \text { ou mais (1), } \\
100-200 \mathrm{~km} \mathrm{(2),50-100} \mathrm{km} \mathrm{(3),} \mathrm{25-50} \mathrm{km} \\
(4), \text { até } 25 \mathrm{~km}(5)\end{array}$ \\
\hline $\begin{array}{l}\frac{1}{0} \\
\frac{0}{0}\end{array}$ & comerctallzaçao & & $\begin{array}{l}\text { Condições de escoamento de produtos } \\
\text { (estradas, etc.) }\end{array}$ & Ótima (3), boa (2), ruim (1) \\
\hline 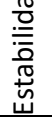 & & & $\begin{array}{l}\text { Tempo necessário para escoar produtos (levar } \\
\text { ao mercado comprador): até a cidade mais } \\
\text { próxima }\end{array}$ & $\begin{array}{l}\text { Até } 1 \text { hora ( } 3) \text {, até } 2 \text { horas ( } 2) \text {, três horas } \\
\text { ou mais (1) }\end{array}$ \\
\hline & Gênero & & Homens e mulheres trabalham no sistema & Homem (1), mulher (1), duas opções (2) \\
\hline & Escolaridade & & $\begin{array}{l}\text { Nível de escolaridade dos responsáveis pelo } \\
\text { SAF }\end{array}$ & Básico (1), médio (2), superior (3) \\
\hline$\frac{0}{0}$ & Emprego & & $\begin{array}{l}\text { Emprega outras pessoas para ajudar no SAF e } \\
\text { outras atividades do agroecossistema }\end{array}$ & Sim (1), não (0) \\
\hline$\frac{\frac{\pi}{0}}{\frac{0}{3}}$ & $\begin{array}{l}\text { Grau } \\
\text { solidariedade }\end{array}$ & & $\begin{array}{l}\text { Cooperação, colaboração entre agricultores, } \\
\text { troca de sementes }\end{array}$ & $\begin{array}{l}\text { Não (0), participa de mutirões (1), troca de } \\
\text { sementes (1), compartilha experiências }\end{array}$ \\
\hline
\end{tabular}




\begin{tabular}{|c|c|c|c|}
\hline & & & (1), somatória (2 ou 3) \\
\hline \multirow{3}{*}{ 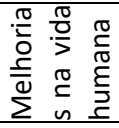 } & Qualidade de vida & Melhoria na qualidade de vida após o SAF & $\operatorname{Sim}(1)$, não (0) \\
\hline & Alimentação & O SAF contribui à alimentação familiar & $\operatorname{Sim}(1)$, não (0) \\
\hline & Conforto laboral & Aumento do conforto do trabalho com os SAFs & $\operatorname{Sim}(1)$, não (0) \\
\hline \multirow[b]{6}{*}{ 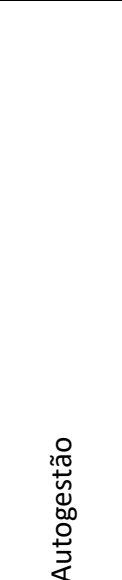 } & Equipamentos & $\begin{array}{l}\begin{array}{l}\text { Disponibilidade de equipamentos } \\
\text { instalações }\end{array} \\
\end{array}$ & Baixa (1), média (2), alta (3) \\
\hline & $\begin{array}{l}\text { Disponibilidade } \\
\text { hídrica }\end{array}$ & Disponibilidade de água & $\begin{array}{l}\text { Aumento (3), normalidade (2), diminuiu } \\
\text { ou diminui em algum período do ano mais } \\
\text { que em anos anteriores (1) }\end{array}$ \\
\hline & Irrigação & Utiliza irrigação & Não (2), $\operatorname{sim}(1)$ \\
\hline & Infraestrutura & $\begin{array}{l}\text { Acesso a infraestrutura básica (moradia, água } \\
\text { encanada, luz, saneamento, estradas, cercas, } \\
\text { etc.) }\end{array}$ & Ótimo (3), bom (2), ruim (1) \\
\hline & Recursos endógenos & Recursos locais aproveitados para produção & Ótimo (3), bom (2), ruim (1) \\
\hline & $\begin{array}{l}\text { Grau de adequação } \\
\text { ao manejo }\end{array}$ & $\begin{array}{l}\text { Em que medida o manejo dos sistemas } \\
\text { produtivos é adequado para manter } \\
\text { produtividade das espécies carro-chefe e } \\
\text { funções ambientais, como ciclagem, sombra, } \\
\text { etc. }\end{array}$ & Ótimo (3), bom (2), ruim (1) \\
\hline
\end{tabular}

*Intepretação do pH baseado no livro: Recomendações para o uso de corretivos e fertilizantes em Minas Gerais - 5a Aproximação.

\section{Organização dos dados}

Os indicadores foram sintetizados em matrizes, com seus respectivos pesos. Como os pesos variam em escala e unidade de medida, alguns de 0 a 4 (exemplo: percentual de culturas agrícolas em relação ao total de plantas do SAF), outros de 0 a 1 (exemplo: o SAF contribui para economia familiar), de 1 a 5 (exemplo: proximidade da cidade mais próxima), ou ainda em porcentagem (tamanho do SAF em relação à propriedade), entre outros, foi necessária a padronização dos dados.

Optou-se pelo método de padronização da porcentagem de linha, onde todos os pesos passam a representar o percentual ocupado em relação a soma da linha que ocupam na matriz pela equação a seguir:

$$
P n(\%)=\left(P n_{i} * 100\right) / \Sigma_{\text {linha }} \quad \text { Equação (1) }
$$

Em que $P n(\%)$ representa o peso em porcentagem de um determinado indicador em cada sistema para determinada linha da matriz; $P n_{i}$ é o peso do indicador dentro da linha, em valores originais; $\Sigma$ linha é o somatório de todos os pesos da linha em seus valores originais. Deste modo, todos os pesos assumiram a mesma unidade de medida (\%), passando a variar de $0 \%$ a $100 \%$ Análise gráfica.

A próxima etapa consistiu na representação gráfica dos indicadores, corroborando com a afirmação de Costa et al. (2012) e Galván-Miyoshi (2008) de que a apresentação deve ser realizada graficamente para a maior facilidade de interpretação pelo público em geral. Desta forma, o layout escolhido gráfico escolhido foi em formato de ameba, ou radar, conforme a recomendação de Masera et al. (2000) e, utilizando os valores médios de cada SAF para todos os atributos.

As interpretações dos desempenhos dos SAFs em relação aos indicadores se deram através da criação de um índice socioambiental para cada atributo (Equação 2):

$$
S A F_{\text {imed }}{ }^{*}\left(A_{\text {indicadores }} / \Sigma_{\text {indicadores }}\right) \quad \text { Equação (2) }
$$

Em que $S A F_{\text {imed }}$ é a média aritmética obtida pelo SAF em um respectivo atributo, Aindicadores representa a quantidade de indicadores inseridas nesse respectivo atributo, $\Sigma_{\text {indicadores }}$ representa a quantidade total de indicadores avaliados, nesse estudo foram 45. Desta forma, dentro de cada atributo, um SAF assume um índice socioambiental (ex.: Índice Socioambiental de Produtividade). Ao final os índices obtidos por cada sistema, para os diferentes atributos, foram somados para se obter o Índice Socioambiental Geral (ISG).

Com base nos desempenhos nos índices socioambientais e nos valores médios obtidos por cada SAF nos atributos, foram resumidos textualmente os fatores positivos e as fragilidades de cada sistema. 


\section{Análise de agrupamento}

Para verificar a similaridade ou a distância entre os sistemas agroflorestais estudados, foi realizada uma análise de agrupamento hierárquico. Essa técnica é utilizada para agrupar dados mediante o grau de similaridade ou dissimilaridade entre eles, envolvendo duas etapas, a primeira diz respeito a adoção de uma medida de distância e a segunda no estabelecimento de um método de agrupamento para formação dos grupos. Como medida de similaridade entre os sistemas agroflorestais foi empregada a distância euclidiana, tradicionalmente empregada em estudos de temática semelhante. O método de agrupamento foi o de Ward, uma técnica de agrupamento que é baseada na variância geral e na variância entre agrupamentos.

\section{RESULTADOS E DISCUSSÃO}

\section{Índice Socioambiental Geral e análise gráfica do desempenho dos SAFs}

O Índice Socioambiental Geral (ISG) é resultado do somatório dos índices obtidos pelos sistemas agroflorestais para cada atributo. Os SAFs com maior ISG podem ser considerados os sistemas com melhores fatores socioambientais.

O ISG variou entre de 7,63 no SAF01 a 14,67 no SAF02. O SAF01 se destacou no atributo autogestão, mas em todos os outros atributos o sistema apresentou um baixo desempenho, diferentemente do SAF02 que se destacou com bons resultados em todos os atributos (Tabela 2).

Tabela 2: Índice socioambiental geral (ISG) para os sistemas agroflorestais avaliados. PRODU=Produtividade;

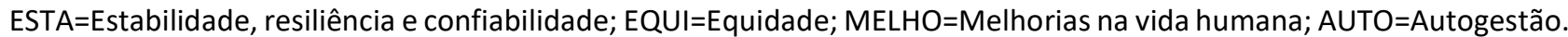
ISG=somatório dos índices obtidos por cada sistema.

\begin{tabular}{lllllll}
\hline Sistema & PRODU & ESTA & EQUI & MELHO & AUTO & ISG \\
\hline SAF01 & 0,79 & 4,12 & 0,89 & 0,00 & 1,83 & 7,63 \\
SAF02 & 4,09 & 6,21 & 1,42 & 1,01 & 1,94 & 14,67 \\
SAF03 & 3,03 & 6,46 & 1,42 & 1,01 & 1,59 & 13,51 \\
SAF04 & 3,43 & 6,64 & 0,98 & 1,01 & 1,76 & 13,81 \\
SAF05 & 2,39 & 6,81 & 1,42 & 1,01 & 1,48 & 13,10 \\
SAF06 & 2,47 & 5,03 & 0,70 & 1,01 & 1,52 & 10,73 \\
SAF07 & 4,79 & 4,67 & 0,76 & 0,63 & 1,49 & 12,34 \\
SAF08 & 3,45 & 6,75 & 1,30 & 1,01 & 1,72 & 14,22 \\
\hline Total (atributo) & 24.44 & 46.67 & 8.89 & 6.67 & 13.33 & 100.00 \\
\hline
\end{tabular}

Os índices socioambientais obtidos refletem o desempenho médio de cada sistema agroflorestal na análise. No atributo Produtividade, as menores médias foram observadas para o SAF01 $(3,663)$, SAF06 (10,14) e SAF05 (10,17), as maiores médias são do SAF07 $(18,66)$, SAF02 (16.73) e SAF04 $(14,25)$, SAF08 e SAF03 com 13,63 e 12,77, respectivamente. Para Estabilidade, resiliência e confiabilidade, SAF01, SAF05 e SAF07 apresentaram as menores médias, ambos abaixo de 12, para todos os demais sistemas os valores médios foram superiores a 13, com destaque ao SAF06 com 14,07.

Em Equidade, o SAF02 e o SAF03 obtiveram o mesmo desempenho, com valor médio de 16, o SAF08 teve média 14,61, todos os outros sistemas obtiveram valores abaixo de 11 . O SAF01 e o SAF07 obtiveram as piores médias para Melhorias da vida humana, o primeiro com 0 e o segundo com 9,3, para os outros SAFs os valores foram iguais $(15,08)$. Em Autogestão, o SAF03, SAF05, SAF06 e SAF07 obtiveram médias abaixo de 12, o SAF02 obteve a maior média (Figura 2). 


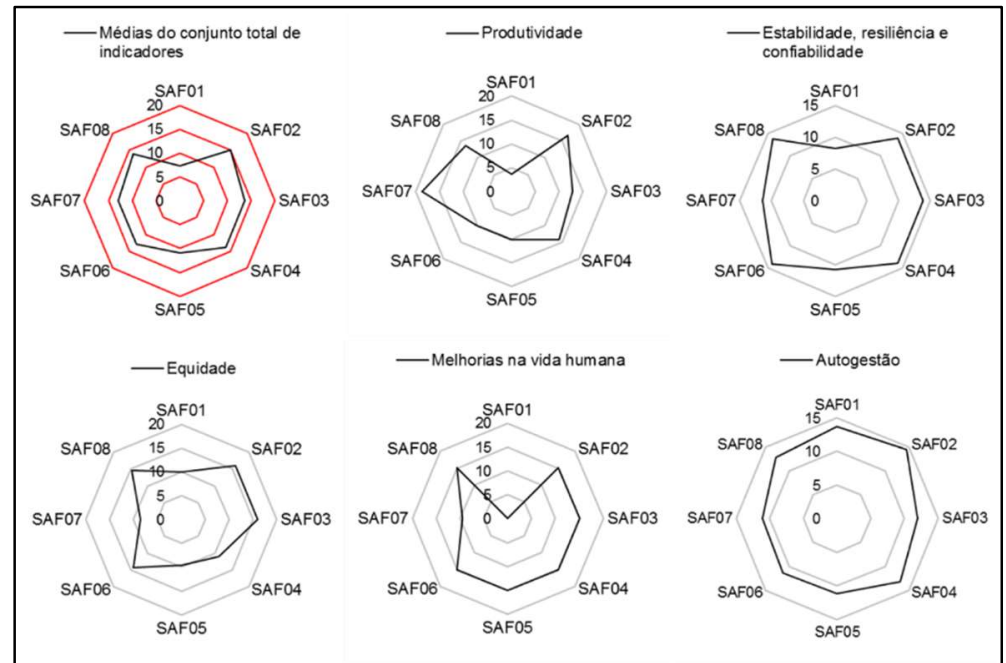

Figura 2: Desempenho médio dos sistemas agroflorestais para cada atributo avaliado.

\section{Análise de agrupamento}

Ocorreu a formação de quatro grupos de sistemas agroflorestais, obtidos a partir da proximidade de valores entre eles (Figura 5).

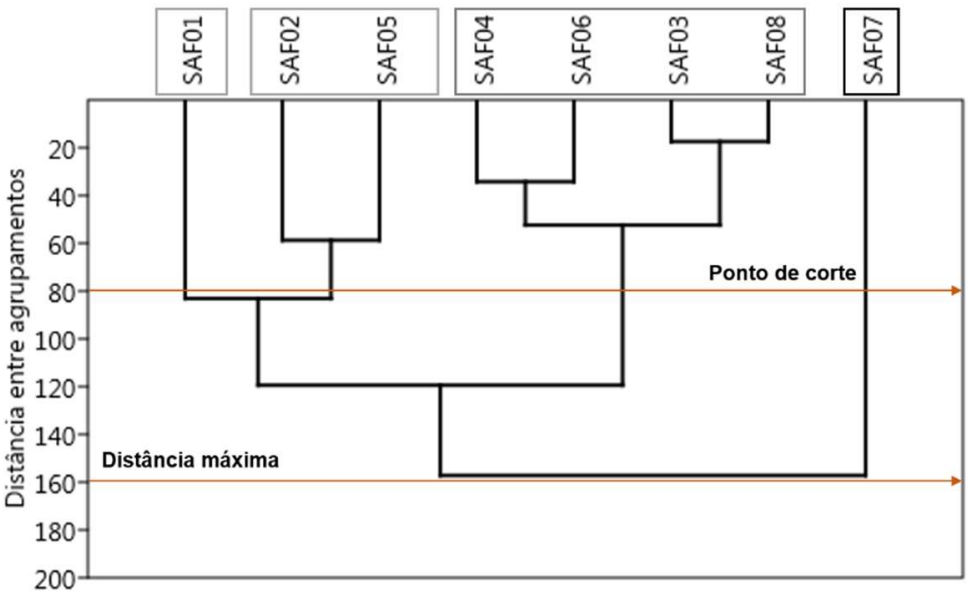

Figura 1: Dendrograma apresentando formação de agrupamento para os SAFs avaliados.

O coeficiente de correlação cofenética (CCC) foi de 0,80 , indicando uma baixa distorção entre a matriz original dos coeficientes de distancias euclidianas dos SAFs avaliados e a matriz cofenética resultante do processo de agrupamento. Estes resultados demonstraram uma boa confiabilidade no método de agrupamento utilizado e, consequentemente, no dendrograma gerado. Dentro de cada agrupamento se inseriram os SAFs com características similares e entre os agrupamentos aqueles que apresentaram características mais distintas.

O primeiro agrupamento com um único sistema agroflorestal, composto pelo SAF01, é justificado principalmente pelos baixos desempenhos desse sistema nos atributos de Produtividade, Estabilidade, resiliência e confiabilidade, Equidade, Melhorias na vida humana, apresentando as menores médias observadas entre os diferentes sistemas agroflorestais avaliados. No atributo Produtividade, o SAF01 não recebeu peso em seis dos onze indicadores; no atributo Estabilidade, resiliência e confiabilidade, não recebeu peso em sete dos indicadores; para o atributo Equidade, esse sistema não recebeu peso em um dos três 
indicadores. Para os três indicadores do atributo Melhorias na vida humana, esse sistema não apresentou peso.

O segundo agrupamento, formado por SAF02 e SAF05 é explicado pelo fato dos dois terem se destacado em alguns indicadores em relação aos demais sistemas avaliados. A principal característica desses dois SAFs são os tamanhos dos sistemas em relação à área do agroecossistema, no SAF02 o sistema agroflorestal ocupa $33,3 \%$ da área total do estabelecimento, o SAF03 ocupa $2,67 \%$, todos os demais ocupam menos de $2 \%$. O percentual de arbóreas nativas em relação ao total de espécies arbóreas também foi mais expressivo nesses dois SAFs, o pH do solo foi considerado bom, thes rendendo maior peso no indicador dessa temática. Quatro agroecossistemas empregam pessoas externas para trabalhar nos SAFs ou em outras atividades, dentre eles estão o SAF02 e SAF05.

O terceiro agrupamento, constituído por SAF03, SAF04, SAF06 e SAF08, é explicado principalmente pela proximidade entre as médias obtidas para os atributos Produtividade, Estabilidade, resiliência e confiabilidade, Melhorias na vida humana e Autogestão. Também são sistemas que ocupam menos de $2 \%$ da área total do agroecossistema, o percentual de arbóreas nativas em relação ao total de espécies arbóreas é menor que $75 \%$ em todos eles, apresentam mais de $50 \%$ de culturas agrícolas em relação ao total de plantas, a proximidade com fragmentos florestais para esses quatro SAFs varia entre $20 \mathrm{~m}$ e $45 \mathrm{~m}$. As agroflorestas SAF03, SAF04 e SAF08 possuem certificação de produção orgânica, fato que também pode ter contribuído para reuni-los em um mesmo grupo.

O SAF07 formou um agrupamento isolado, isso ocorreu devido alguns fatores como por não possuir certificação de produção orgânica, também por ser o único local onde a terra é única fonte de renda do núcleo gestor. Ainda por não terem sido identificadas espécies arbóreas no SAF, pelo fato de ainda serem mudas, sendo o único a receber peso zero para esse indicador. $\mathrm{O}$ pH do solo foi considerado baixo $(4,4) \mathrm{o}$ que rendeu também um peso baixo para esse indicador; o núcleo gestor não tem e nem teve acesso à políticas públicas de aquisição de alimentos (ex.: PNAE); as condições de escoamento de produtos são ruins (como estradas não pavimentadas e esburacadas); é o único entre todos os SAFs em que o proprietário não tem nível superior de ensino; ainda não obteve aumento de conforto no trabalho proporcionado pelo sombreamento das árvores do sistema, pelo fato de ainda serem mudas. 0 agroecossistema também tem pouca disponibilidade de equipamentos e instalações para o manejo, bem como para o processamento de produtos oriundos do sistema.

\section{CONCLUSÕES}

Até a data de realização do presente estudo não foram encontradas publicações científicas que empregam indicadores de sustentabilidade ou socioambientais de sistemas agroflorestais multiestrata no Cerrado goiano, por esse motivo essa pesquisa desempenha um importante papel por meio da tentativa de sintetizar indicadores que ajudaram a elucidar, não em sua completude, mas uma parte da complexidade existente entre os fatores ambientais e sociais que interagem com esses sistemas agrícolas diversificados.

Nessa primeira análise, através de uma adaptação do método MESMIS, foi possível observar a 
eficiência produtiva dos SAFs e do agroecossistema, bem como alguns fatores que influenciam no potencial de comercialização dos produtos oriundos desses sistemas. Foi possível identificar, através dos indicadores, o perfil dos agricultores que cultivam SAFs no Cerrado goiano, um pouco das características organizacionais dos agroecossistemas, também qual a contribuição desses sistemas para a qualidade de vida dos agricultores.

Assim como a agroecologia, a avaliação de agroecossistemas por meio de indicadores deve ser mutável e aceitar a multiplicidade de diferentes abordagens metodológicas, rompendo com o determinismo mecanicista que direciona a experimentação agronômica. Deste modo, o presente estudo se constitui como uma experiência inicial de se trabalhar com indicadores socioambientais em SAFs no Cerrado goiano, para as próximas pesquisas serão necessários mais testes para a incorporação de critérios que abranjam de forma mais completa a complexidade dos agroecossistemas, tentando elencar fatores similares e diferentes entre eles, bem como alguns indicadores mais generalistas que possam ser replicados em outros sistemas com diferentes componentes e práticas de manejo diversas.

\section{REFERÊNCIAS}

COSTA, A. M; TORRES-MANSO, F.; TIBÉRIO, T.. Sustainability diagnosis of an agroforestry system. Regional Science Inquiry Journal, v.4, n.2, p.111-124, 2012.

DANIEL, O.. Definição de indicadores de sustentabilidade para sistemas agroflorestais. Tese (Doutorado em Ciência Florestal) - Universidade Federal de Viçosa, Viçosa, 2000.

DUBOC, E.. Sistemas agroflorestais e o cerrado. In: FALEIRO, F. G.. Savanas: desafios e estratégias para o equilíbrio entre sociedade, agronegócio e recursos naturais. Planaltina: Embrapa Cerrados, 2008. p.964-985.

EMBRAPA. Empresa Brasileira de Pesquisa Agropecuária. Manual de métodos de análises de solos. 2 ed. Rio de Janeiro: Embrapa Solos, 2011.

FERNANDES, P. A.; PESSÔA, V. L. S.. O Cerrado e suas atividades impactantes: uma leitura sobre o garimpo, a mineração e a agricultura mecanizada. Observatorium: Revista Eletrônica de Geografia, v.3, n.7, p.19-37, 2011.

FILGUEIRAS, T. S.; BROCHADO, A. L.; NOGUEIRA, P.E.; GUALA, G. F.. Caminhamento: um método expedito para levantamentos florísticos qualitativos. Cadernos de Geociências, v.12, p.39-43, 1994.

FONTORA, Y.; NAVES, F.. Movimento agroecológico no Brasil: A construção da resistência à luz da abordagem neogramsciana. Organização \& Sociedade, v.23, n.77, p.329347, 2016. DOI: https://doi.org/10.1590/1984-9230778

GALVÁN-MIYOSHI, Y.. Integración de indicadores en la evaluación de sustentabilidad: de los índices agregados a la representación multicriterio. In: ASTIER, M.. Evaluación de sustentabilidad. Un enfoque dinámico y multidimensional. Valencia: Fundación Instituto de Agricultura Ecológica y Sustentable, 2008. p.95-115.

MASERA, O.; ASTIER, M.; LÓPEZ-RIDAURA, S.. Sustentabilidad ymanejo de recursos naturales: el marco de evaluación MESMIS. Ciudad de México: Mundi-Prensa, 2000.
MICCOLIS, A.; PENEIREIRO, F. M.; MARQUES, H. R.; VIEIRA, D. L. M.; ARCO-VERDE, M. F.; HOFFMANN, M. R.; REHDER, T.; PEREIRA, A. V. B.. Restauração Ecológica com Sistemas Agroflorestais: como conciliar conservação com produção. Opções para Cerrado e Caatinga. Brasília: Instituto Sociedade, População e Natureza, 2016.

MONTELLANO, F. H. A. W.. Diseño de una metodología para evaluar Sistemas Agroforestales en zonas secas dentro del Proyecto Isabel, Modulo Pairumani. Monografía (Licenciatura en Ingeniería Ambiental) - Universidad Católica Boliviana, Cochabamba, 2013.

NAIR, P. K. R.. An introduction to agroforestry. Dordrecht: Kluwer Academic, 1993.

NAIR, P. K. R.. Classification of Agroforestry Systems. Agroforestry Systems, v.3, p.97-128, 1985. DOI: https://doi.org/10.1007/BF00122638

NAIR, P. K. R.. State-of-the-art of agroforestry systems. Forest Ecology and Management, v.45, p.5-29, 1991. DOI: https://doi.org/10.1016/0378-1127(91)90203-8

NASCIMENTO, J. S.; FRANCO, F. S.; LOPES, P. R.. Duas décadas de floresta: indicadores de sustentabilidade em sistemas agroflorestais no município de Cananéia, SP. Cadernos de Agroecologia, v.13, n.1, p.1-6, 2018.

PENEIREIRO, M.; RODRIGUES, F. Q.; BRILHANTE, M. O.; LUDEWIGS, T.. Apostila do educador agroflorestal Introdução aos sistemas agroflorestais: um guia técnico. Rio Branco: UFAC, 2002.

REFLORA. Herbário Virtual. Consulta pública do herbário virtual. Rio de Janeiro, 2016.

SANTAMARIA, L. S.; RAMIREZ-HERNANDEZ, O.. Evaluación de agroecosistemas mediante indicadores de sostenibilidad en San José de las Lajas, provincia de Mayabeque, Cuba. Luna Azul, n.44, p.120-152, 2017. 
SCHROTH, G.; MOTA, M. S. S.. Agroforestry: Complex Multiestrata Agriculture. In: VAN ALFEN, N.. Encyclopedia of Agriculture and Food Systems. 2 ed. San Diego: Elsevier, 2014. p.185-207.

SILVA NETO, B.. Sistemas Agrários e Agroecologia: a dinâmica da agricultura e as condições para uma transição agroecológica no município de Porto Xavier (RS). Revista Brasileira de Agroecologia, v.9, n.2, p.15-29, 2014.
TESSIER, L.; BIJTTEBIER, J.; MARCHAND, F.; BARET, P. . A mixed method approach to characterize and explain the pursuit of agroecological principles at Flemish beef farms. In: EUROPEAN IFSA SYMPOSIUM, 13; PROCEEDINGS OF EUROPEAN IFSA SYMPOSIUM. Anais. Viena: IFSA, 2018. p.115.

TORQUEBIAU, E.. Are tropical agroforestry home gardens sustainable?. Agriculture, Ecosystems and Environment, v.41, p.189-207, 1992. DOI: https://doi.org/10.1016/01678809(92)90109-0

A CBPC - Companhia Brasileira de Produção Científica (CNPJ: 11.221.422/0001-03) detém os direitos materiais desta publicação. Os direitos referem-se à publicação do trabalho em qualquer parte do mundo, incluindo os direitos às renovações, expansões e disseminações da contribuição, bem como outros direitos subsidiários. Todos os trabalhos publicados eletronicamente poderão posteriormente ser publicados em coletâneas impressas sob coordenação da Sustenere Publishing, da Companhia Brasileira de Produção Científica e seus parceiros autorizados. Os (as) autores (as) preservam os direitos autorais, mas não têm permissão para a publicação da contribuição em outro meio, impresso ou digital, em português ou em tradução. 